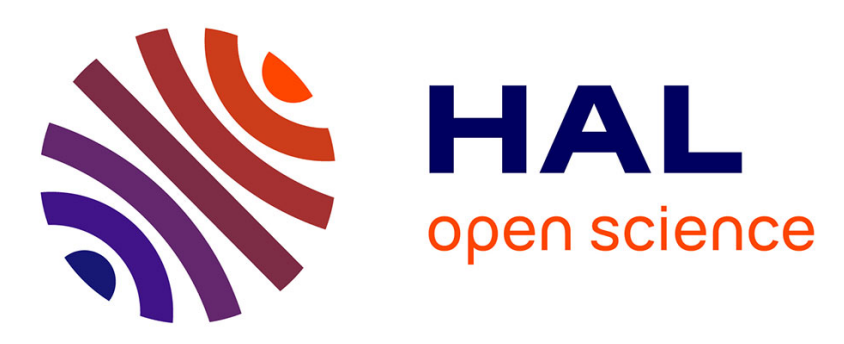

\title{
Temporal tracking of 3D coronary arteries in projection angiograms
}

Guy Shechter, Frédéric Devernay, Ève Coste-Manière, Elliot R. Mcveigh

\section{To cite this version:}

Guy Shechter, Frédéric Devernay, Ève Coste-Manière, Elliot R. Mcveigh. Temporal tracking of 3D coronary arteries in projection angiograms. Medical Imaging, SPIE, 2002, San Diego, United States. $10.1117 / 12.467204$. inria-00262390

\section{HAL Id: inria-00262390 \\ https://hal.inria.fr/inria-00262390}

Submitted on 11 Mar 2008

HAL is a multi-disciplinary open access archive for the deposit and dissemination of scientific research documents, whether they are published or not. The documents may come from teaching and research institutions in France or abroad, or from public or private research centers.
L'archive ouverte pluridisciplinaire HAL, est destinée au dépôt et à la diffusion de documents scientifiques de niveau recherche, publiés ou non, émanant des établissements d'enseignement et de recherche français ou étrangers, des laboratoires publics ou privés. 


\title{
Temporal tracking of 3D coronary arteries in projection angiograms
}

\author{
Guy Shechter $^{a b c}$, Frédéric Devernay ${ }^{b}$, Eve Coste-Manière ${ }^{b}$, Elliot R. McVeigh ${ }^{c a}$ \\ ${ }^{a}$ Johns Hopkins University, 720 Rutland Ave., Baltimore, USA \\ ${ }^{b}$ INRIA, 2004 rte des Lucioles, Sophia Antipolis, FRANCE \\ ${ }^{c}$ NHLBI, 10 Center Drive, Bethesda, USA
}

\begin{abstract}
A method for 3D temporal tracking of a 3D coronary tree model through a sequence of biplane cineangiography images has been developed. A registration framework is formulated in which the coronary tree centerline model deforms in an external potential field defined by a multiscale analysis response map computed from the angiogram images. To constrain the procedure and to improve convergence, a set of three motion models is hierarchically used: a 3D rigid-body transformation, a 3D affine transformation, and a 3D B-spline deformation field. This 3D motion tracking approach has significant advantages over 2D methods: (1) coherent deformation of a single 3D coronary reconstruction preserves the topology of the arterial tree; (2) constraints on arterial length and regularity, which lack meaning in 2D projection space, are directly applicable in 3D; and (3) tracking arterial segments through occlusions and crossings in the projection images is simplified with knowledge of the 3D relationship of the arteries. The method has been applied to patient data and results are presented.
\end{abstract}

Keywords: Coronary Arteries, Motion Tracking, 3D, Registration, X-ray Angiography

\section{INTRODUCTION}

As early as 1971, Kong $^{1}$ proposed quantifying regional myocardial performance using the 3D motion of coronary artery bifurcations as observed in $\mathrm{X}$-ray angiograms. Over the last ten years, researchers have begun using reconstructions of the entire 3D coronary tree to describe the motion and function of the left ventricle epicardial surface. ${ }^{2-4}$ These $3 \mathrm{D}+\mathrm{t}$ (three-dimensional plus time) reconstructions of the coronary tree can provide quantitative information about vessel lengths, volume and flow measurements ${ }^{5,6}$ at different phases of the cardiac cycle, as well as providing quantitative descriptions of stenoses morphology. ${ }^{7}$ Taken together, the motion and morphology of the coronary arteries are important determinants in cardiovascular disease diagnosis, therapy planning and outcome assessment.

Traditionally, the temporal evolution of the 3D coronary tree is recovered by tracking the motion of the centerlines in the 2D projection images, and subsequently, independently reconstructing the 3D representation at later time frames. Optical flow, ${ }^{8}$ binary image elastic registration, ${ }^{9}$ Kalman snakes, ${ }^{10}$ and local space search and graph minimization ${ }^{11}$ methods have been used to track the vascular motion in the projection images. However, these $2 \mathrm{D}$ tracking methods have several limitations. For example, reconstruction ambiguities can arise when tracking vessels into regions of multiple vessel overlap, which may lead to structural differences between the $3 \mathrm{D}$ models reconstructed at different time frames. In addition, imposing true regularization constraints on the shape and length of the deforming 3D structure is not possible when tracking in 2D.

Making the transition to 3D, Ruan ${ }^{12}$ proposed combining optical flow derived displacement information from two projection images to displace a given coronary tree model in $3 \mathrm{D}$ space. Bascle ${ }^{13}$ described the use of deformable 3D B-spline curves for segmenting objects in a stereo image pair, an idea which has been applied to the coronary arteries by Cañero. ${ }^{14}$ In this paper, we further develop the 3D approach for tracking a coronary tree model through a biplane cineangiography image sequence. We propose a 3D registration framework for tracking the motion of a 3D coronary artery model, guided by an external force field derived from the projection images, and internal forces which regularize the coronary morphology and motion in 3D. We use a coarse-to-fine motion

For further information and/or correspondence: shechter@bme.jhu.edu

This paper will be published in Medical Imaging 2002: Image Processing, SPIE Proceedings Vol. 4684 and is made available as an electronic preprint with permission of SPIE. One print or electronic copy may be made for personal use only. Systematic or multiple reproduction distribution to multiple locations via electronic or other means, duplication of any material in this paper for a fee or for commercial purposes, or modification of the content of the paper are prohibited. 
tracking approach to match the deforming coronary model to the images of the cineangiogram sequence using a global 3D rigid body transformation, a global 3D affine transformation, and a local 3D B-spline (B-solid ${ }^{15}$ ) deformation field.

\section{METHODS}

\subsection{Imaging protocol}

All images were acquired using a Philips BH5000 biplane cardiovascular angiography system. For each of 5 patients, a biplane sequence of images of the opacified left coronary artery tree during 3-4 cardiac cycles was acquired during suspended respiration. The position of the imaging arms was not constrained, and varied from patient to patient to maximize separation of the arteries on the projection images. The frame rate of each imaging plane was 15 frames per second.

Following the patient's departure from the imaging suite, the imaging system was reoriented to the same configuration used during the acquisition of patient data, and images of a calibration grid and a phantom were acquired. The calibration grid, a plastic plate $(23 \times 23 \times 0.2 \mathrm{~cm})$ of radio-opaque beads $(1 \mathrm{~mm}$ diameter, $1 \mathrm{~cm}$ horizontal and vertical spacing) was imaged while affixed to the image intensifier. The plastic rectangular phantom $(4.9 \times 6.73 \times 2.49 \mathrm{~cm})$ containing nine metal beads $(3.18 \mathrm{~mm}$ diameter $)$ was imaged at a location approximating the patient's heart.

\subsection{Geometric Distortion Correction and Validation of Imaging Geometry}

Images acquired using standard $\mathrm{X}$-ray equipment typically suffer from two independent geometric distortions: (1) the curved face of the image intensifier generates a pincushion distortion, which is observable as a stretching in the periphery of images; and (2) the interaction of the Earth's magnetic field with the electrons in the image intensifier unit generates an imager-orientation dependent S-shaped distortion. Images of the calibration grid affixed to the face of the image intensifier are used to compensate for these distortions.

The sub-pixel location of the grid's beads are automatically identified using a combination of greylevel thresholding, morphological operations, and an intensity weighted centroid calculation. Subsequently, the undistorted bead locations over the entire image are hypothesized from the observed bead positions in the center of the distorted image, using the assumption that the geometric distortion is minimized at the image center (Figure 1a). Two polynomials are used to independently model the distorted $x_{d}$ and $y_{d}$ bead coordinates as a function of the undistorted bead position $\left(x_{u}, y_{u}\right)$ :

$$
x_{d}=\sum_{i=0}^{N} \sum_{j=0}^{M} P_{i, j}\left(x_{u}\right)^{i}\left(y_{u}\right)^{j} \quad y_{d}=\sum_{i=0}^{N} \sum_{j=0}^{M} Q_{i, j}\left(x_{u}\right)^{i}\left(y_{u}\right)^{j}
$$

where $P_{i, j}$ and $Q_{i, j}$ are the coefficients of the degree $N, M$ bi-polynomials. Using the matched distorted and undistorted bead positions over the entire image, a system of linear equations is constructed and solved by QR factorization to recover the coefficients $P_{i, j}$ and $Q_{i, j}$.

In practice, fifth degree bi-polynomials are used to calculate the distorted position corresponding to an ideal pixel position, and the corrected image is generated by bilinear interpolation of the original distorted image at that position. For five different orientations of the image intensifier, the degree five bi-polynomials were able to fit the geometric distortion with an average 2D norm error of less than 0.2 pixels, and a sub-pixel maximum error.

This approach is able to reliably correct high-order geometric distortion, but because the undistorted positions of the beads are computed from the central imaged beads, this method cannot recover low-order distortions affecting the entire image, which may include bulk 2D translation and rotation. Reconstruction of the phantom, and comparison with its known geometry, is used to validate the imaging geometry, as provided in the image headers, and to recover any global image distortions.

A non-linear least squares solver is used to find the in-plane rotations of the two imaging planes and the 2D inplane translation of one imaging plane, which minimize the reprojection errors of the phantom (Figure 1b) and 
the 3D error of the reconstructed phantom with respect to the known phantom geometry. After optimization of these parameters, for each of the four imaging configurations used to acquire patient data, the RMS reprojection error of the phantom reconstruction was less than 0.4 pixels, and the RMS 3D error was less than $0.6 \mathrm{~mm}$.

(a)

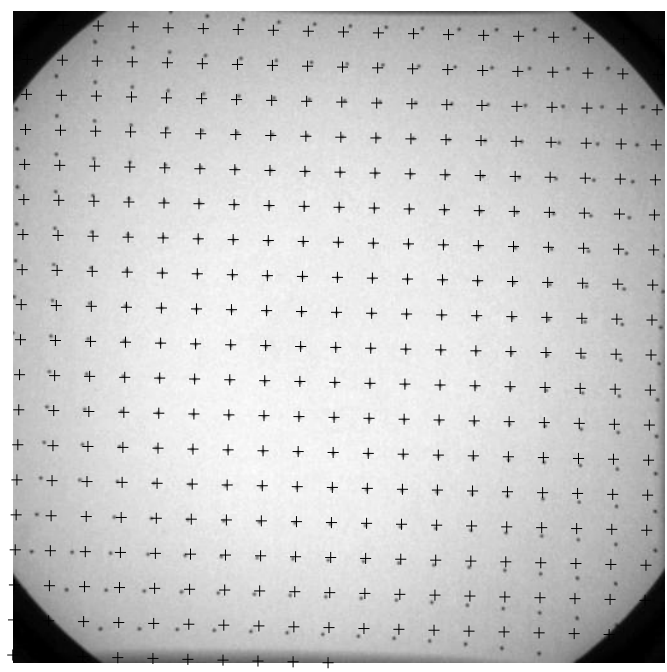

(b)

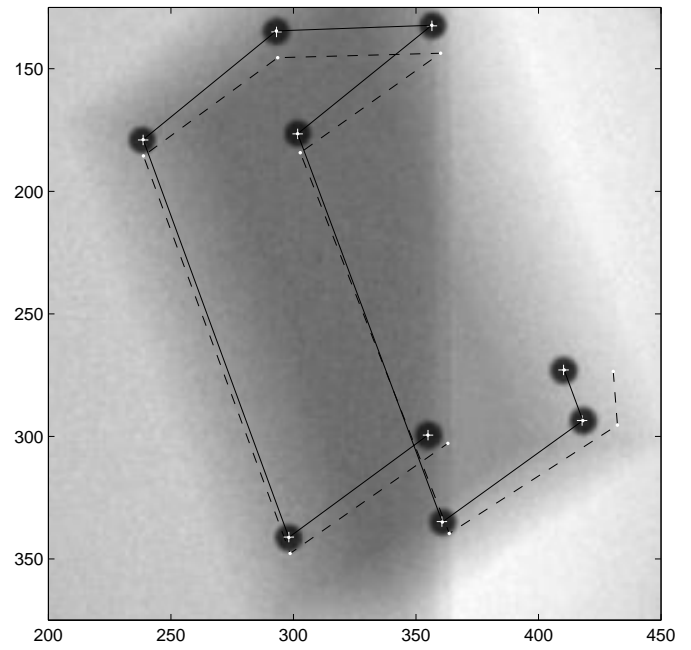

Figure 1. (a) The positions of the undistorted beads $(+)$ calculated from and superimposed on an image of the calibration grid $\left(0^{\circ} \mathrm{LAO} / \mathrm{RAO}, 0^{\circ}\right.$ Cranial/Caudal, $1058 \mathrm{~mm}$ SID). (b) Reprojections of a reconstructed phantom before (dashed line) and after (solid line) correcting for low-order image distortion.

(a)



(b)

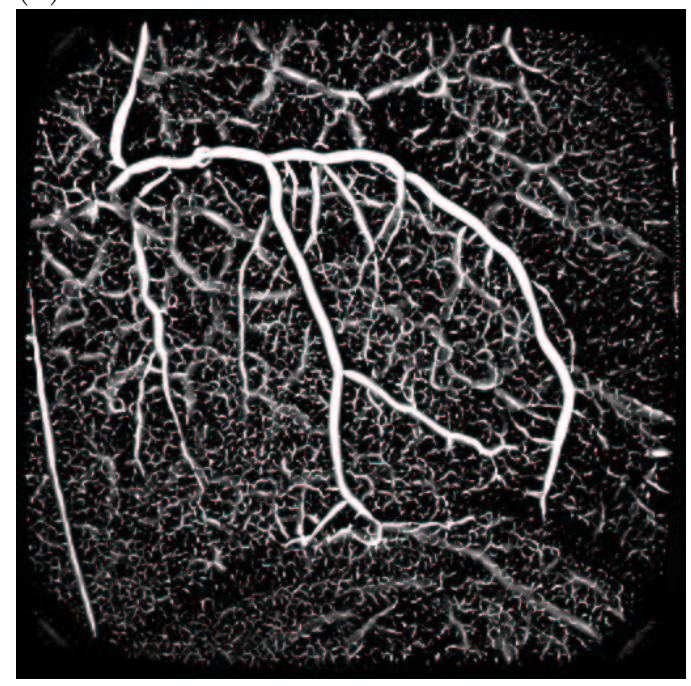

Figure 2. An example projection image (a) of the left coronary tree $\left(45^{\circ} \mathrm{RAO}, 0^{\circ}\right.$ Cranial/Caudal, $1044 \mathrm{~mm}$ SID) and its corresponding maximum multiscale response map (b). The multiscale analysis retains rectilinear structures and removes planar structures such as the diaphragm and ribs. 
(a)



(c)

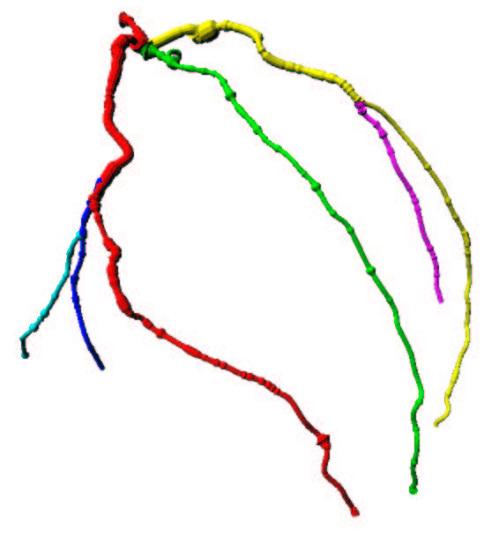

(b)
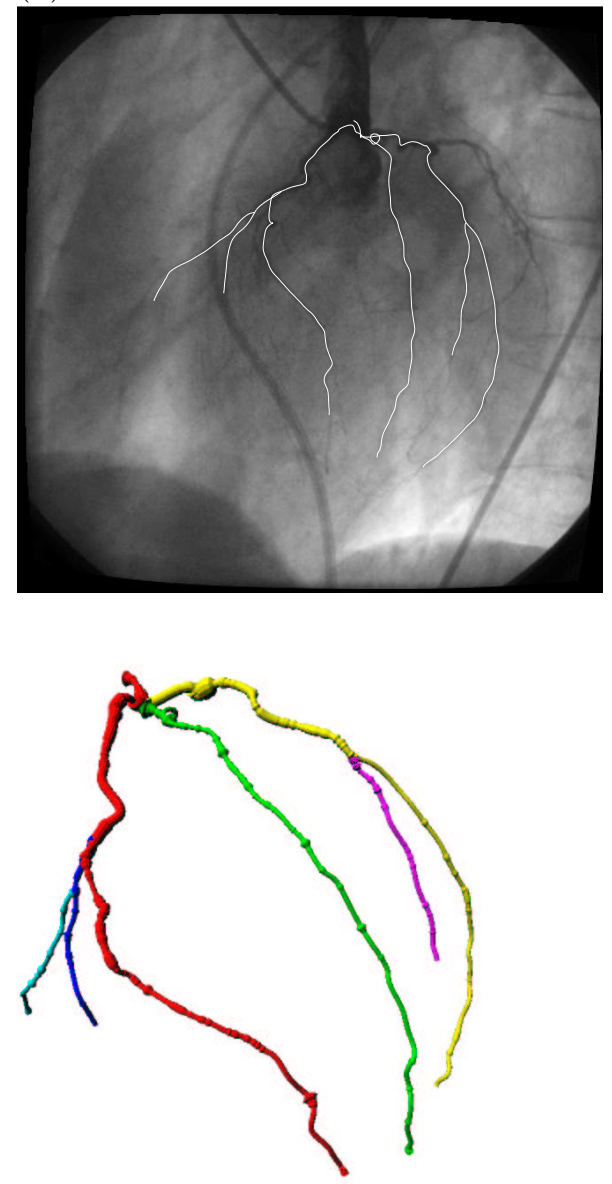

Figure 3. RAO (a) and LAO (b) projections of the left coronary tree at end diastole. In (c), a wide-eyed stereogram of the reconstructed arteries.

\subsection{Reconstruction of a 3D Coronary Tree}

Our method for 3D temporal tracking of the motion of the coronaries through a biplane cineangiogram image sequence requires an a priori 3D model of the coronary tree (Figure 3). For each patient, this model is reconstructed at one time frame at the end of diastole using an extension of the technique developed by Mourgues. ${ }^{16}$ The angiogram images are first processed with a set of multiscale filters. The maximal response over the set of filters is stored as the maximum response map for that image, and by thresholding, provides a noisy segmentation of the coronary centerlines (Figure 2). A semi-interactive tool is used to manually draw the arteries on the projection images, using the maximum multiscale response map to constrain the procedure.

The 2D centerlines are converted to a B-spline representation and are hierarchically organized. The epipolar constraint is used to define point to point correspondences along the lengths of the drawn arteries using a dynamic programming graph search strategy. Finally, a discrete 3D model of the coronary centerlines is reconstructed by computing the intersections of rays connecting matched projection points with their $\mathrm{X}$-ray source in a least-squares sense.

To reduce storage and computational requirements for tracking the motion of the coronaries, the discrete representation of the tree is converted to a parametric representation. The use of $\mathrm{B}$-spline basis functions allows for a more compact description of the arterial curves, and provides intrinsic smoothness to the curves. 
The discrete coronary tree is first decomposed into arterial segments $\left\{A_{0}, A_{1}, \ldots\right\}$. A segment, $A_{k}$, is defined as the ordered set of points describing a unique part of the coronary tree such that (1) the segment includes and is terminated at both extremes by a bifurcation point, or (2) includes a bifurcation point at one extreme and at the other extreme a leaf or root point of the tree. Thus, each $A_{k}=\left\{\mathbf{q}_{0}, \mathbf{q}_{1}, \ldots, \mathbf{q}_{m_{k}}\right\} \in \mathbb{R}^{3}$ contains $m_{k}+1$ discrete points representing an arterial segment. To each ordered set of points, $A_{k}$, we fit an approximating spline curve $\mathbf{C}_{k}(u) \in \mathbb{R}^{3}$, using a chord length parameterization of $A_{k}$, so that

$$
\left\{\mathbf{C}_{k}(u) \mid u \in[0,1]\right\} \approx A_{k} .
$$

Specifically, $\mathbf{C}_{k}(u)$ is a $3 \mathrm{D}$ B-spline curve defined as

$$
\mathbf{C}_{k}(u)=\sum_{i=0}^{n_{k}-1} B_{i, p}(u) \mathbf{V}_{i}^{k}
$$

where the $\left\{\mathbf{V}_{i}\right\} \in \mathbb{R}^{3}$ are the $n_{k}$ control points, and the $\left\{B_{i, p}\right\}$ are the $p$ th degree B-spline basis functions. Using the length $(m+1)$ non-periodic and non-uniform knot vector, where $m=n_{k}+p+1$, and

$$
\mathbf{U}_{k}=\{\underbrace{0, \ldots, 0}_{p+1}, u_{p+1}, \ldots, u_{m-p-1}, \underbrace{1, \ldots, 1}_{p+1}\},
$$

the values at the ends of the curve are constrained so that

$$
\begin{gathered}
\mathbf{C}_{k}(0)=\mathbf{V}_{0}^{k}=\left(\mathbf{q}_{0} \in A_{k}\right) \\
\mathbf{C}_{k}(1)=\mathbf{V}_{n_{k}}^{k}=\left(\mathbf{q}_{m_{k}} \in A_{k}\right) .
\end{gathered}
$$

This strategy allows us to maintain $C^{0}$ continuity between a parent artery, $A_{k}$, and a child artery, $A_{j}$, by forcing them to share a control point:

$$
\mathbf{C}_{k}(1)=\mathbf{C}_{j}(0) \quad \text { if } \quad \mathbf{V}_{n_{k}}^{k}=\mathbf{V}_{0}^{j}
$$

In practice, we use cubic $\mathrm{B}$-spline basis functions $\left(p=3, C^{2}\right.$ continuity) for all artery segments, and maintain $C^{0}$ continuity across the bifurcations.

\subsection{Motion Models}

The goal of our motion tracking algorithm is to recover the transformations $M^{t}: \mathbb{R}^{3} \rightarrow \mathbb{R}^{3}$, which map any point $\mathbf{q}$ on the coronary tree at time $t_{0}$, to the point's position at time $t_{0}+t$,

$$
\mathbf{q}^{t}=M^{t}(\mathbf{q}) \text {. }
$$

We adopt a hierarchical coarse-to-fine approach for recovering the transformation $M^{t}$. In this section, we describe the three motion models we use to recover the motion of the coronaries: a 3D rigid body transformation, a 3D affine transformation, and a B-solid deformation field.

\subsubsection{D Rigid Body Transformation}

The class of 3D rigid body transformations describes motion of a non-deforming object in $\mathbb{R}^{3}$ using six degrees of freedom: three angles of rotation, and three translation parameters. The transformation of a point $\mathbf{q}$ at time $t_{0}$ to its position $\mathbf{q}^{t}$ at time $t_{0}+t$ can be written as

$$
M_{R}^{t}(\mathbf{q})=\left[\begin{array}{lll|l} 
& R_{t} & & T_{t} \\
& & & \\
\hline 0 & 0 & 0 & 1
\end{array}\right]\left[\begin{array}{c}
\mathbf{q} \\
\hline 1
\end{array}\right]
$$

where in $\mathbb{R}^{3}, R_{t}$ is a $3 \times 3$ rotation matrix with Euler angles $\left(\theta_{t}, \phi_{t}, \psi_{t}\right)$ and $T_{t}=\left(\Delta x_{t}, \Delta y_{t}, \Delta z_{t}\right)^{T}$ is a $3 \mathrm{D}$ translation vector. The use of Euler angles is justified since the magnitude of the rotations are expected to be small. Because B-splines are invariant under rigid transformation, we can write the rigid body transformation functional of the curve $\mathbf{C}(u)$ from equation (1) as

$$
M_{R}^{t}[\mathbf{C}]=\sum_{i=0}^{n-1} B_{i, p}(u) M_{R}^{t}\left(\mathbf{V}_{i}\right) .
$$




\subsubsection{D Affine Transformation}

The class of 3D affine transformations describes motion of a non-rigid object in $\mathbb{R}^{3}$ using twelve degrees of freedom. The transformation of a point $\mathbf{q}$ at time $t_{0}$ to its position $\mathbf{q}^{t}$ at time $t_{0}+t$ can be written as

$$
M_{A}^{t}(\mathbf{q})=\left[\begin{array}{lll|c} 
& & & T_{t} \\
& & & \\
\hline 0 & 0 & 0 & 1
\end{array}\right]\left[\begin{array}{c}
\mathbf{q} \\
\hline 1
\end{array}\right]
$$

where in $\mathbb{R}^{3}, A_{t}$ is a $3 \times 3$ matrix with nine free parameters $\left(a_{11}, a_{12}, a_{13}, \ldots, a_{33}\right)$ and $T_{t}=\left(\Delta x_{t}, \Delta y_{t}, \Delta z_{t}\right)^{T}$ is a $3 \mathrm{D}$ translation vector. The matrix $A_{t}$ can be decomposed into three rotations, three scale factors, and three shears. Because B-splines are invariant under affine transformation, we can write the $3 \mathrm{D}$ affine transformation functional of the curve $\mathbf{C}(u)$ from equation (1) as

$$
M_{A}^{t}[\mathbf{C}]=\sum_{i=0}^{n-1} B_{i, p}(u) M_{A}^{t}\left(\mathbf{V}_{i}\right) .
$$

\subsubsection{B-solid Transformation}

A B-solid transformation is used to model local deformations of the coronary arteries. The B-solid structure is the dimension three analog of the one dimensional B-spline curve, and the two dimensional B-spline tensor product surface. We define the 3D B-spline deformation field $D^{t}: \mathbb{R}^{3} \rightarrow \mathbb{R}^{3}$ which, for a point on the coronary tree at $t_{0}$, provides the $3 \mathrm{D}$ displacement vector to the point's position at time $t_{0}+t$. For a point $\mathbf{q}=\left(q_{x}, q_{y}, q_{z}\right) \in \mathbb{R}^{3}$ at time $t_{0}$, we can write

$$
D^{t}(\mathbf{q})=\sum_{i=0}^{n_{i}-1} \sum_{j=0}^{n_{j}-1} \sum_{k=0}^{n_{k}-1} B_{i, p}\left(q_{x}\right) B_{j, p}\left(q_{y}\right) B_{k, p}\left(q_{z}\right) \mathbf{S}_{i j k}^{t}
$$

where the $\left\{\mathbf{S}_{i j k}^{t}\right\} \in \mathbb{R}^{3}$ are the $n_{i} \times n_{j} \times n_{k}$ control points, and the $\left\{B_{\cdot, p}\right\}$ are the $p$ th degree B-spline basis functions. The control point density is directly proportional to the amount of local deformation that can be represented by the B-solid. By using cubic B-spline basis functions $(p=3), C^{2}$ continuity of the deformation field is maintained over the B-solid volume. Each B-spline basis function is defined on a uniform knot vector over the coronary tree range in that dimension. Finally, the B-solid transformation of a point $\mathbf{q}$ at time $t_{0}$ to its position $\mathbf{q}^{t}$ at time $t_{0}+t$ can be written as

$$
M_{B}^{t}(\mathbf{q})=\mathbf{q}+D^{t}(\mathbf{q}) .
$$

We approximate the B-solid transformation functional for the curve $\mathbf{C}(u)$ from equation (1) as

$$
M_{B}^{t}[\mathbf{C}]=\sum_{i=0}^{n-1} B_{i, p}(u) M_{B}^{t}\left(\mathbf{V}_{i}\right) .
$$

\subsection{Motion Tracking}

A registration framework is adopted to recover the set of transformations $\left\{M^{t}: t=1,2, \ldots\right\}$ which register the $3 \mathrm{D}$ coronary tree from time $t_{0}$ with the observed projection images at time $t_{0}+t$. To recover the transformation $M^{t}$, we describe a system composed of a $3 \mathrm{D}$ coronary tree defined at time $t_{0}$, the motion transformation $M^{t}$, and a biplane angiogram image pair at time $t_{0}+t$. To quantify the quality of the transformed coronary tree's fit to the projection images, we define an energy measure for our system,

$$
E_{\text {total }}=-\omega_{1} E_{\text {external }}+\omega_{2} E_{\text {arteries }}+\omega_{3} E_{\text {bsolid }}
$$

which is a weighted sum of an external angiographic-image derived energy term, an internal arterial energy term, and when solving for the B-solid transformation, an internal B-solid energy term. Motion tracking aims 
at recovering the "best" transformation, $M^{t}$, which minimizes the energy functional defined in equation (5) and nulls its derivative taken with respect to the transformation,

$$
\frac{\partial E_{\text {total }}}{\partial M^{t}}=-\omega_{1} \frac{\partial E_{\text {external }}}{\partial M^{t}}+\omega_{2} \frac{\partial E_{\text {arteries }}}{\partial M^{t}}+\omega_{3} \frac{\partial E_{\text {bsolid }}}{\partial M^{t}}=0 .
$$

To solve for $M^{t}$ at time $t_{0}+t$, the rigid transformation, $M_{R}^{t}$, is recovered first, and used to initialize the search for the affine transformation, $M_{A}^{t}$. Then, $M_{A}^{t}$ is used to initialize the recovery of the B-solid deformation field, which is the final transformation $\left(M^{t}=M_{B}^{t}\right)$. Finally, $M^{t}$, the recovered transformation at time $t_{0}+t$, is used to initialize the search for $M^{t+1}$.

\subsubsection{External Energy}

The external energy is calculated using a potential map, $R_{i}$, computed for the projection X-ray angiography image, $I_{i}$, using the multiscale response method described in Section 2.3. For a given motion functional, $M^{t}$, and a set of curves defining the $3 \mathrm{D}$ coronary tree at time $t_{0},\left\{\mathbf{C}_{a}(u)\right\}$, we integrate the values of the potential field of the projected transformed curves along their 3D arc-length and normalize by the length of the coronary tree and the number of projections,

$$
E_{\text {external }}=\left(n_{p} \sum_{a=1}^{n_{a}} L\left[\hat{\mathbf{C}}_{a}\right]\right)^{-1} \sum_{i=1}^{n_{p}} \sum_{a=1}^{n_{a}} \int_{0}^{L\left[\hat{\mathbf{C}}_{a}\right]} R_{i}\left(x_{i}^{a}(s), y_{i}^{a}(s)\right) d s
$$

where $n_{p}$ is the number of projection images, $n_{a}$ is the number of arteries in the $3 \mathrm{D}$ coronary tree model, and the transformed coronary B-spline curve $\hat{\mathbf{C}}_{a}=M^{t}\left[\mathbf{C}_{a}\right]$ is itself a B-spline curve with control points $\hat{\mathbf{V}}_{i}=M^{t}\left(\mathbf{V}_{i}\right)$ (see eqns. 2, 3,4). The functional $L$ calculates the arc-length of a 3D B-spline curve defined over the parametric interval $[0,1]$ by evaluating

$$
L\left[\mathbf{C}_{a}\right]=\int_{0}^{1}\left\|\mathbf{C}_{a}^{\prime}(u)\right\| d u .
$$

Finally, $x_{i}^{a}(u)$ and $y_{i}^{a}(u)$ are the projection coordinates of $\hat{\mathbf{C}}_{a}$ on the $i$ th projection image. Given the $3 \mathrm{x} 4$ projection matrix, $\mathbf{P}^{i}$, which defines the projection of the 3D model coordinates to local image coordinates for image $i$, the projected transformed coronary 3D B-spline can be written in homogeneous coordinates as

$$
\mathbf{P}^{i} M\left[\mathbf{C}_{a}\right]=\mathbf{P}^{i} \hat{\mathbf{C}}_{a}(u)=\sum_{j=0}^{n-1} B_{j, p}(u) \mathbf{P}^{i} \hat{\mathbf{V}}_{j} .
$$

The non-homogeneous coordinates of the projected curve can be expressed as two non-uniform rational Bsplines (NURBS),

$$
\begin{aligned}
& x_{i}(u)= \frac{\sum_{j=0}^{n-1} B_{j, p}(u)\left(P_{1,1}^{i} \hat{V}_{j}^{x}+P_{1,2}^{i} \hat{V}_{j}^{y}+P_{1,3}^{i} \hat{V}_{j}^{z}+P_{1,4}^{i}\right)}{\sum_{j=0}^{n-1} B_{j, p}(u)\left(P_{3,1}^{i} \hat{V}_{j}^{x}+P_{3,2}^{i} \hat{V}_{j}^{y}+P_{3,3}^{i} \hat{V}_{j}^{z}+P_{3,4}^{i}\right)} \\
& y_{i}(u)=\frac{\sum_{j=0}^{n-1} B_{j, p}(u)\left(P_{2,1}^{i} \hat{V}_{j}^{x}+P_{2,2}^{i} \hat{V}_{j}^{y}+P_{2,3}^{i} \hat{V}_{j}^{z}+P_{2,4}^{i}\right)}{\sum_{j=0}^{n-1} B_{j, p}(u)\left(P_{3,1}^{i} \hat{V}_{j}^{x}+P_{3,2}^{i} \hat{V}_{j}^{y}+P_{3,3}^{i} \hat{V}_{j}^{z}+P_{3,4}^{i}\right)}
\end{aligned}
$$

Since the arc-length reparameterization of a B-spline curve can be written as

$$
\begin{aligned}
s(\mathbf{C}, u) & =\int_{0}^{u}\left\|\mathbf{C}^{\prime}(\tilde{u})\right\| d \tilde{u} \\
\frac{d s(\mathbf{C}, u)}{d u} & =\left\|\mathbf{C}^{\prime}(u)\right\|
\end{aligned}
$$

and if we assume that the length of a coronary artery changes minimally, we can make the assumption that $L\left[\hat{\mathbf{C}}_{a}\right] \approx L\left[\mathbf{C}_{a}\right]$ which simplifies equation (6) to

$$
E_{\text {external }}=\left(n_{p} \sum_{a=1}^{n_{a}} L\left[\mathbf{C}_{a}\right]\right)^{-1} \sum_{i=1}^{n_{p}} \sum_{a=1}^{n_{a}} \int_{0}^{1} R_{i}\left(x_{i}^{a}, y_{i}^{a}\right)\left\|\hat{\mathbf{C}}_{a}^{\prime}(u)\right\| d u .
$$




\subsubsection{Arterial Energy}

The internal arterial energy is a regularizing term which prevents large changes in the lengths of the arteries $\left\{\mathbf{C}_{a}(u)\right\}$ due to the transformation $M^{t}$,

$$
E_{\text {internal }}=\sum_{a=1}^{n_{a}}\left[L\left[M^{t}\left[\mathbf{C}_{a}\right]\right]-L\left[\mathbf{C}_{a}\right]\right]^{2}
$$

where $n_{a}$ is the number of arteries in the $3 \mathrm{D}$ coronary tree model, and $\mathrm{L}$ is the arc-length functional defined in equation (7).

\subsubsection{B-solid Energy}

The internal B-solid energy term is used to regularize the values at neighboring control points in the B-spline grid. Its use is based on the assumption that, in general, the motion of the coronaries is spatially continuous. Therefore, the energy of the B-solid structure is defined as a 3D Laplacian operator, and normalized by the number of control points in the B-solid,

$$
E_{\mathrm{bsolid}}=\frac{1}{n_{i} n_{j} n_{k}} \sum_{e=0}^{n_{i}-1} \sum_{f=0}^{n_{j}-1} \sum_{g=0}^{n_{k}-1}\left[\sum_{i=-1}^{1} \sum_{j=-1}^{1} \sum_{k=-1}^{1} \mathbf{w}_{i, j, k} \cdot \mathbf{S}_{e+i, f+j, g+k}^{t}\right]^{2} .
$$

$\mathbf{w}$ is a $9 \times 9 \times 9$ spatial weighting kernel where $\mathbf{w}_{i, j, k}=\left[\begin{array}{lll}w_{i, j, k} & w_{i, j, k} & w_{i, j, k}\end{array}\right]$, and

$$
w_{i, j, k}= \begin{cases}\sqrt{|i|+|j|+|k|}, & \text { if } i, j, k \neq 0 \\ -\sum_{p, q, r=-1}^{1} \sqrt{|p|+|q|+|r|}, & \text { if } i, j, k=0\end{cases}
$$

\subsection{Quantifying the Error of the Recovered Transformation}

After recovering a transformation $M^{t}$, an independent quantitative assessment of its error is computed. Since the true 3D transformation of the coronary tree is not known, the use of a 3D metric is not possible. Instead, we quantify the error in the space of the $2 \mathrm{D}$ projection images.

First, the projection angiogram images at time $t_{0}+t$, are segmented interactively using the semi-automatic multiscale method described in Section 2.3. This set of manually defined points representing the coronary centerlines for projection $i$ is labeled $F_{i}$.

For the transformation $M^{t}$, the transformed coronary tree is computed and sampled in 3D with an arc-length resolution of $1 \mathrm{~mm}$. Each point of the sampled transformed coronary tree is projected onto the angiogram view $i$ using the $3 \times 4$ projection matrix $\mathbf{P}_{i}$. Finally, for each projected point, a $2 \mathrm{D}$ pixel distance is computed to the closest point in $F_{i}$, and converted to millimeters using the known intensifier size and magnification factor for that projection.

During systole, the distal parts of the arteries disappear from the images, probably due to their collapse secondary to high intraventricular pressure. Therefore, any 3D coronary point, whose distance to the closest point in both projection images is greater than 10 pixels, is discarded. Visual inspection is used to confirm that this procedure does not throw out points which truly belong to mistracked arteries.

The reprojection error for $M^{t}$ is computed as the RMS of the 2D norm distances in the biplane views.

\section{RESULTS}

The motion of the coronary arteries was tracked in five patient data sets. The 3D coronary tree models were reconstructed at end-diastole and tracked through one cardiac cycle, which ranged between 9 and 15 frames for the five data sets. For each motion model, the procedure was allowed to converge on a set of smoothed versions of the multiscale response maps, with standard deviations of the Gaussian kernel corresponding to $\sigma=4,2,1$, and 0.5 pixels. Two of the patients required an additional pre-iteration using the rigid motion model with a 

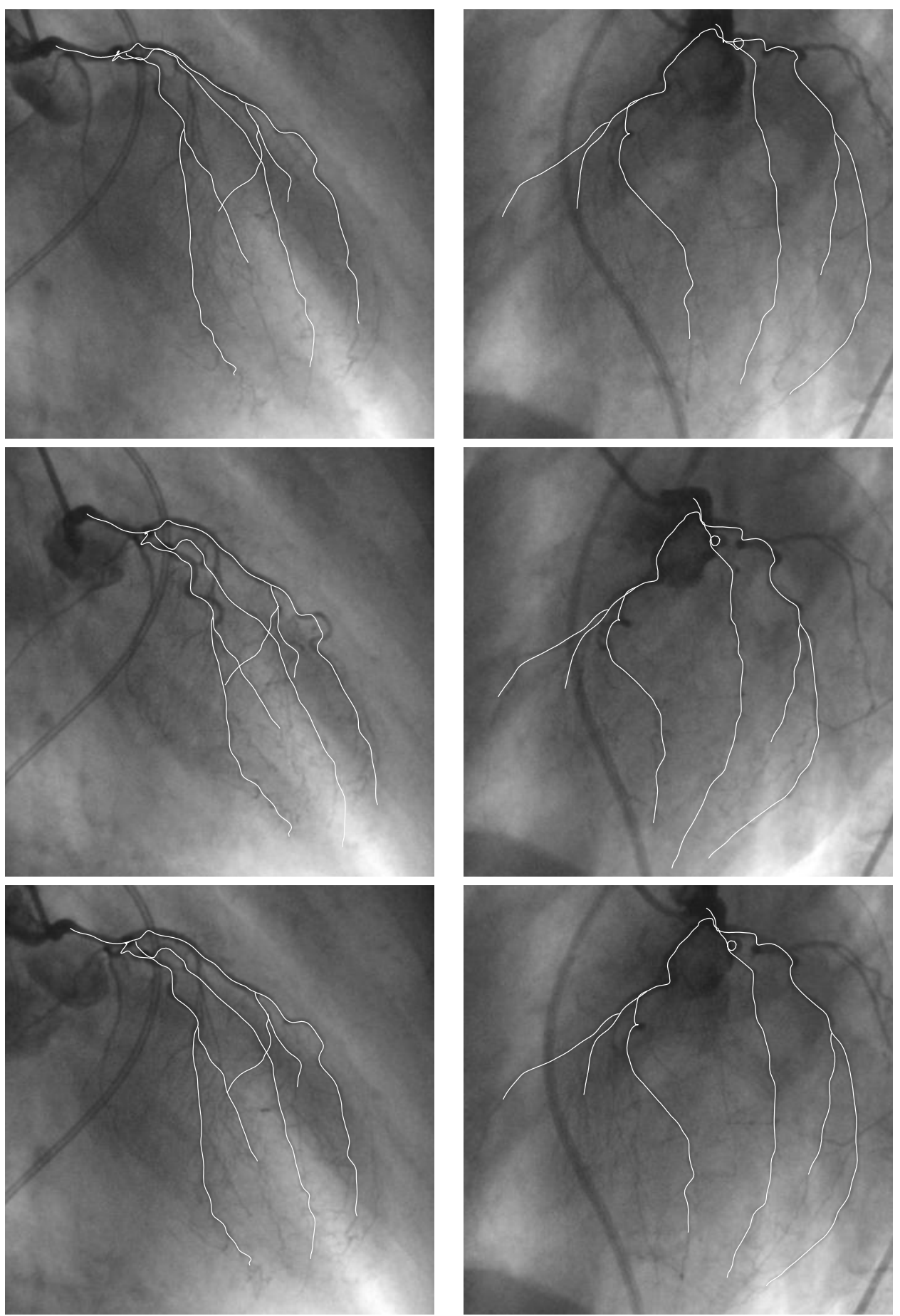

Figure 4. Tracking results for one patient over one cardiac cycle. The biplane image pairs correspond to atrial contraction (top row), systole (middle row) and end-diastole (bottom row). 
(a)

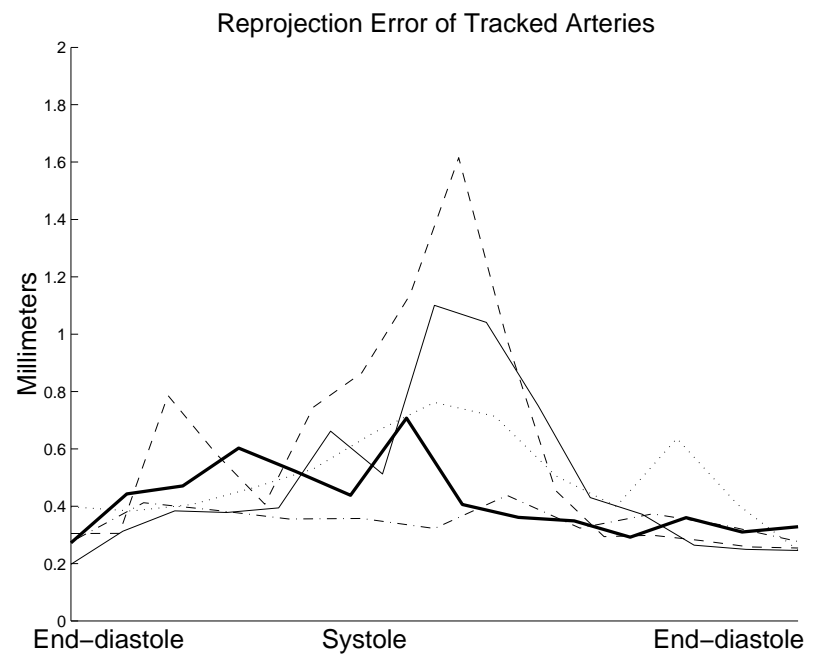

(b)

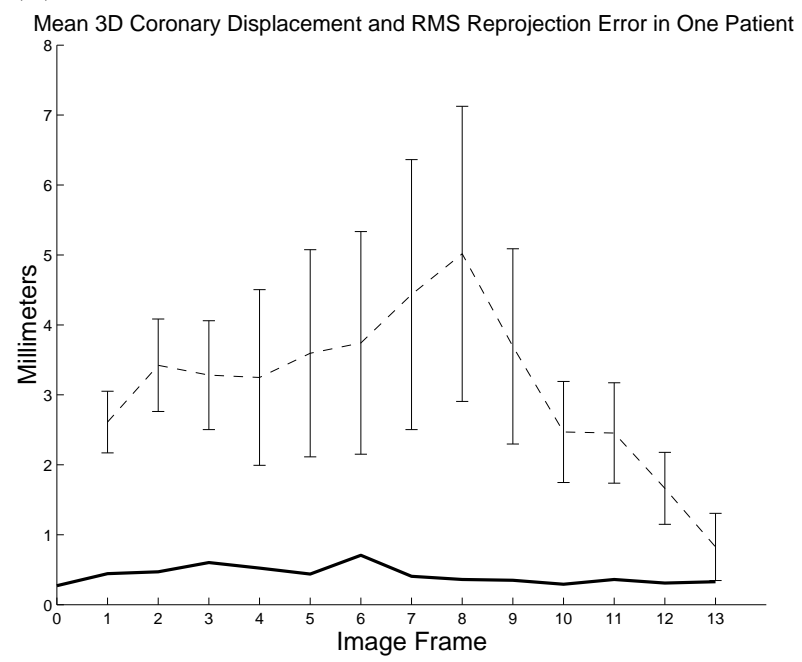

Figure 5. (a) The RMS reprojection errors following tracking through one cardiac cycle in five patient data sets. (b) In one patient, the mean $3 \mathrm{D}$ displacements of the coronary tree (error bars represent one standard deviation) from end-diastole are computed, and shown in comparison to the measured RMS reprojection error.

smoothed potential map using $\sigma=8$ in order to capture the rapid motion of their arteries. An 8x8x8 control point grid was used for the B-solid motion model in all patients. For the affine motion model, the coefficients of equation (5) were: $\omega_{1}=1, \omega_{2}=0.05, \omega_{3}=0$. For the B-solid motion model, the coefficients were: $\omega_{1}=1$, $\omega_{2}=0.001, \omega_{3}=0.0001$.

Tracking results for one patient data set are presented in Figure 4. The recovered motion models are applied to the 3D coronary tree reconstructed at end-diastole, and the result is reprojected on the angiogram images. The biplane image pairs correspond to images during the atrial contraction (top row), during the ventricular contraction (middle row), and at the next end-diastolic phase (bottom row).

The RMS reprojection errors for the tracked arteries of the five patients are presented in Figure 5a. Because of the differing heart rates, the five patients' results are rescaled temporally and plotted over one cardiac cycle. In Figure 5b, the RMS reprojection error for one patient is plotted together with the average magnitude of 3D displacements of points on the coronary tree from end-diastole.

The motion tracking procedure was implemented in MatLaB (Mathworks, Inc.) and the five data sets were processed on a number of Linux computers, with Pentium III processors operating between $750 \mathrm{MHz}$ to $1 \mathrm{GHz}$. The mean running time for motion recovery from one time frame to the next was 155 minutes ( $\sigma=58$ minutes).

\section{DISCUSSION}

The results we have obtained are encouraging, especially because the patient population used for this study has a history of severe cardiac disease, including myocardial infarcts and diffuse coronary artery disease.

Using the method presented in this paper, the motion of the coronary arteries is recovered one frame at a time. The unsmooth reprojection error plots of Figure 5 suggest that the recovered transformation may not capture the temporal regularity typically associated with physiological motion. A logical extension of our method calls for the implementation of temporal continuity of the rigid and affine motion models and the use of four dimensional tensor product B-splines.

However, temporal discontinuities are expected during the cardiac cycle, particularly between the systolic and diastolic phases. This knowledge must be embedded in the temporal regularizing functions, in order to be able to correctly capture rapid motion changes. In fact, a priori knowledge about the coronary motion can be 
used to improve convergence of the tracking procedure, but care must be taken to avoid over-constraining the procedure, which would make recovery of atypical motion more difficult.

Motion tracking with temporal continuity will also provide a framework to overcome inconsistencies due to the non-synchronous nature of the biplane imaging system. To reduce X-ray scatter, the two imaging views are acquired at an interleaved 15 frames per second, instead of the 30 frames per second commonly used for single plane angiography. In this paper, a given biplane image pair is assumed to be acquired synchronously, when there is in fact a 16 millisecond temporal offset between the images. Rapid motion may produce irreconcilable observations in the two views, which would interfere with convergence of the motion tracking for that time frame. Using temporally continuous motion models will allow each image to be assigned its correct temporal index.

The RMS reprojection errors of the five patients show a characteristic pattern of increasing during systole and decreasing into diastolic relaxation. We discuss two different hypotheses for explaining the increased error during systole. One hypothesis is that localized deformation of the coronary arteries during systole, especially in patients with highly tortuous vasculature, cannot be adequately described using the $8 \times 8 \times 8$ control point grid used for the B-solid motion model in these experiments. This density was chosen based on experiments for recovery of myocardial motion from tagged MR images, ${ }^{17}$ but may not be sufficient for describing the coronary deformation. However, the use of more dense control point grids was avoided because of the prohibitive computation times given the current implementation. In lieu of increasing the control point density, a deformable contour approach may be considered as a final step for capturing the local conformational changes.

Another hypothesis for explaining the higher systolic reprojection errors derives from the observed disappearance of the distal ends of the coronary arteries during systole. Physiologically, extravascular compression of the intra-myocardial arteries can lead to their collapse or to a reversal of flow in the coronaries during systole. The disappearance of the arteries may cause two problems. First, the lack of an imaged coronary for the motion model to track, introduces ambiguity and may lead to tracking of other structures or of the arteries being subjected to only the globally recovered motion of the coronary tree. The coronary disappearance problem also complicates the RMS reprojection error computation, where a distinction must be systematically made to retain closest point matches that correspond to mistracked arteries, and to exclude closest point matches that are erroneous due to the inability to manually segment the distal ends of the arteries in the systolic images.

\section{CONCLUSION}

We have presented a method for 3D temporal tracking of the coronary arteries in a sequence of biplane angiograms. A coarse-to-fine approach using three motion models is used to recover the motion from one time frame to the next. The method has been applied to five patient data sets, and encouraging results have been obtained and quantified.

\section{ACKNOWLEDGMENTS}

The authors thank Dr. Arshed Quyyumi, Dr. Khaled Abdul-Nour, Bill Schenke, Stan White, Gloria Zalos, and other members of the Cardiac Catheterization Section of the National Heart Lung and Blood Institute, NIH, for their assistance in obtaining patient data, and Jason Lee for construction of the calibration grid and phantom.

Special thanks to Christophe Blondel and Hervé Delingette for numerous discussions and helpful suggestions. 


\section{REFERENCES}

1. Y. Kong, J. Morris, and H. McIntosh, "Assessment of regional myocardial performance from biplane coronary cineangiograms," The American Journal of Cardiology 27, pp. 529-537, May 1971.

2. A. Young, P. Hunter, and B. Smaill, "Estimation of epicardial strain using the motions of coronary bifurcations in biplane cinéangiography," IEEE Transactions on Biomedical Engineering 39(5), pp. 526-531, 1992.

3. G. Coppini, M. Demi, P. Marracini, and A. L'Abbate, "3-D heart motion from X-ray angiography," IEEE Computers in Cardiology, pp. 71-74, 1995.

4. J. Puentes, C. Roux, M. Garreau, and J. Coatrieux, "Dynamic feature extraction of coronary artery motion using DSA image sequences," IEEE Transactions on Medical Imaging 17(6), pp. 857-871, 1998.

5. N. Guggenheim, P. Dorsaz, P. Doriot, C. Suilen, F. Chappuis, and W. Rutishauser, "3D determination of the intravascular volume and flow of coronary arteries," Internation Journal of Bio-Medical Computing 35, pp. 13-23, 1994.

6. A. Wahle, E. Wellnhofer, I. Mugaragu, H. Sauer, H. Oswald, and E. Fleck, "Assessment of diffuse coronary artery disease by quantitative analysis of coronary morphology based upon 3-D reconstruction from biplane angiograms," IEEE Transactions on Medical Imaging 14, pp. 230-241, June 1995.

7. T. Saito, M. Misaki, K. Shirato, and T. Takishima, "Three-dimensional quantitative coronary angiography," IEEE Transactions on Biomedical Imaging, pp. 768-777, Aug. 1990.

8. J. Meunier, M. Bourassa, M. Bertrand, M. Verreault, and G. Mailloux, "Regional epicardial dynamics computed from coronary cineangiograms," pp. 307-310, 1990.

9. B. Tom, S. Efstratiadis, and A. Katsaggelos, "Motion estimation of skeletonized angiographic images using elastic registration," IEEE Transactions on Medical Imaging 13(3), pp. 450-460, 1994.

10. R. Curwen, A. Amini, J. Duncan, and F. Lee, "Tracking vascular motion in X-ray image sequences with Kalman snakes," Computers in Cardiology, pp. 109-112, 1994.

11. M.-P. Dubuisson-Jolly, C. Liang, and A. Gupta, "Optimal polyline tracking for artery motion compensation in coronary angiography," in International Conference in Computer Vision, pp. 414-419, 1998.

12. S. Ruan, A. Bruno, R. Collorec, and J. Coatrieux, "3D motion and reconstruction of coronary networks," pp. 2048-2049, 1992.

13. B. Bascle, Contributions and Applications of Deformable Models in Computer Vision. PhD thesis, University of Nice-Sophia Antipolis, 1994.

14. C. Cañero, P. Radeva, R. Toledo, J. Villanueva, and J. Mauri, "3D curve reconstrucion by biplane snakes," in Proceddings of the International Conference on Pattern Recognition, pp. 563-566, 2000.

15. P. Radeva, A. Amini, and J. Huang, "Deformable B-solids and implicit snakes for 3D localization and tracking of SPAMM MRI data," International Journal on Computer Vision and Image Understanding 66, pp. 163-178, May 1997.

16. F. Mourgues, F. Devernay, G. Malandain, and È. Coste-Manière, "3D+t modeling of coronary artery tree from standard non simultaneous angiograms," in Lecture Notes in Computer Sciences, Medical Image Computing and Computer-Assisted Intervention (MICCAI2001), 2208, pp. 1320-1322, Springer-Verlag, Oct. 2001.

17. C. Ozturk and E. McVeigh, "Four dimensional B-spline based motion analysis of tagged cardiac MR images," in Proceedings of SPIE Medical Imaging, 3660, pp. 46-56, Feb. 1999. 